\title{
Characteristics of Transient Eddies During Ural Blocking Events With Different Lifetime in Winter
}

\author{
Jinyu Zhang ${ }^{1}$, Yan $L^{2,3 *}$, Zhilan Wang ${ }^{1}$, Yu Zhang ${ }^{1}$ and Yao $L u^{2}$ \\ ${ }^{1}$ Institute of Arid Meterology, China Meteorological Administration, Lanzhou, China, ${ }^{2}$ College of Atmospheric Sciences, Lanzhou \\ University, Lanzhou, China, ${ }^{3}$ Key Laboratory of Semi-Arid Climate Change, Ministry of Education, Lanzhou University, Lanzhou, \\ China
}

Ural blocking high (hereafter UB) is one of the most important weather systems influencing the weather and climate of China, with its onset and development closely connected with transient eddies. Herein, we classified the wintertime UB events during 1979-2015 into three types according to their lifetime, i.e., short, medium, and long lifetime UB events and further analyzed the characteristics of momentum and heat fluxes transported by transient eddies during the three types of UB events. The results show that the eddy momentum and heat transport by transient eddies over the Ural regions is strong, while the westerly is weak, favorable for the establishment and maintenance of the UB. Before the onset of all the UB events there are enhanced lower-level temperature disturbances, decelerated

OPEN ACCESS

Edited by:

Bo Huang,

Norwegian University of Science and

Technology, Norway

Reviewed by:

Yaocun Zhang,

Nanjing University, China

Yipeng Guo,

Nanjing University, China

*Correspondence:

Yan $\mathrm{Li}$

liyanlz@lzu.edu.cn

Specialty section:

This article was submitted to Interdisciplinary Climate Studies,

a section of the journal

Frontiers in Earth Science

Received: 15 January 2022

Accepted: 04 February 2022

Published: 02 March 2022

Citation:

Zhang J, Li Y, Wang Z, Zhang Y and Lu Y (2022) Characteristics of Transient Eddies During Ural Blocking Events With Different Lifetime in Winter.

Front. Earth Sci. 10:855634. doi: 10.3389/feart.2022.855634 westerly and convergence of transient momentum and heat fluxes. After the onset of the UB, the upper-level jet stream is accelerated and eddy flux convergence is enhanced. Furthermore, it is found that the duration and position of convergence could be one of important factors determining the lifetime of UB event. The long-time strong convergence of transient eddies favors the maintenance of UB. During long UB events, the polar jet and the convergence of eddy momentum fluxes have the strongest intensity and northward shifted position. The convergence is further strengthened until Day +2 and weakened since Day +7 , favorable for the long-time maintenance of UB. There is convergence on Day -3 of both short and medium UB events, which disappears on Day +4 during short events, while strengthened after the onset of medium events, leading to a long-time maintenance of UB. Furthermore, among the three types of UB events, the upward propagation of wave activity and $\mathrm{E}-\mathrm{P}$ flux divergence are the strongest and most long-lasting during long UB events, while the weakest and shortest during short UB events, which can also verify strong atmospheric baroclinicity and long-lasting strong convergence of transient eddies are favorable for long duration of the UB.

Keywords: Ural blocking, transient eddy, transport of momentum and heat, E-P flux, lifetime of blocking high

\section{INTRODUCTION}

Blocking high is one of the weather systems with anomalous meridional development of mid-high latitude atmospheric circulations, the onset and collapse of which can lead to meridional exchange of large-scale air parcel and heat (e.g., Charney and Devore 1979; Mokhov et al., 2013). This can induce drastic changes in the large-scale, or even hemispheric, atmospheric circulation and further lead to 
weather and climate extremes, or even severe weather disasters. Ural region is one of the regions with the largest blocking frequency over Northern Hemisphere in winter (e.g., Lupo and Smith 1995; Masato et al., 2013). The Ural blocking (hereafter UB) is important for the weather and climate in China. The onset, development and collapse of UB are closely linked to the Meiyu rainfall over the Jianghuai region in China and the establishment of UB is also an important precursor for the outbreak of cold spells over East Asia (Ding and Johnny 2005; Takaya and Nakamura 2005; Bueh et al., 2011; Luo et al., 2016a). Therefore, it is necessary to investigate the main factors influencing the establishment of wintertime UB and the dynamic and thermodynamic characteristics during the onset of UB events, which can not only provide theoretic basis for understanding the mechanism of the UB development, but also help improve our understanding of the causes of the weather and climate extremes associated with UB events.

Many previous studies have investigated the underlying mechanism responsible for the development of blocking high: Yeh (2010) studied the onset and decay of blocking high from a perspective of energy dispersion. Huang and Zou (1989) found that basic mean flow provides energy for eddies during the establishment of blocking high, leading to a rapid strengthening of eddies. Luo 2003; Luo et al., 2016b) and Shi and Nakamura 2020 revealed the close connection between the development and maintenance of UB and the propagation of Rossby waves. Luo and Zhang (2020a) considered that the local wave activity fluxes are favorable for the establishment of blocking high during its growth phase. Many studies found that the energy for the establishment and maintenance of blocking high mainly comes from baroclinic energy conversion of effective potential energy from basic zonal flow and non-linear interaction between waves, with vorticity transport by transient eddies as a manifestation of the latter (Luo et al., 2014; Ma and Liang 2017; Li et al., 2020). Similarly, the convergence and divergence of transient vorticity and heat flux play a major role in the onset and maintenance of the wintertime blocking high (Nakamura et al., 1997; Luo and Chen 2005; Athar and Lupo 2010). Xu and Jin (2011) diagnosed the influence of transient eddy fluxes on anomalous blockings and found that anti-cyclonic flow is strengthened due to wave-mean flow interaction. Shi and Wang (2021) found that high-frequency transient eddies are favorable for the maintenance of wintertime UB through barotropic energy conversion.

As a large-scale weather system, the lifetime of blocking high is 5-7 days on average, with the longest events up to 20 days (Diao et al., 2006; Yao et al., 2017). Blocking highs with different lifetimes have different influences on the weather and climate (Ye et al., 2015). Some previous studies focused on the potential factors influencing blocking lifetime. Kong and $\mathrm{Hu}$ (2014) also declared that long-duration UB events could occur more frequently during negative stratospheric northern annular mode (hereafter NAM) events. Ye et al. (2015) found that the stronger the blocking highs over Atlantic and Pacific, the more possibly long UB events could occur. Furthermore, it is found that the meridional gradient of potential vorticity can significantly influence the lifetime and intensity of UB events
(Luo et al., 2019; Luo and Zhang 2020b). In addition, Luo (2003) demonstrated that the pre-existing synoptic-scale eddies are a key driver of the spatio-temporal evolution of blocking. The negative phase of North Atlantic Oscillation (NAO) can enhance the planetary-scale split-jetflow prior to blocking onset, through the interaction with upstream synoptic-scale waves, favorable for the establishment and long duration of blocking high (Luo and chen 2005; Wan and Luo 2009) and then the enhanced Eurasian cold extremes (Feng et al., 2019). However, so far, there are few studies on the influence of transient waves on the life cycle of the UB events.

From the above-mentioned previous studies, it can be found that transient eddy, as an important component of atmospheric waves, is important for the maintenance of blocking high. However, the potential influence of transient eddies on the lifetime of UB event still remains unclear. In this study, we attempted to address the following two questions: 1) what is the role of transient eddies in the development of UB events in boreal winter? 2) What are the characteristics and possible impact of transient eddies on UB events with different lifetimes? The rest of the paper is organized as follows: Section $\mathbf{2}$ describes the data and methodologies. Section 3 describes the characteristics of atmospheric circulation evolution during the UB events. Sections $\mathbf{4}$ and $\mathbf{5}$ analyze the characteristics of eddy momentum and heat transported by transient eddies during the UB events with different lifetimes, respectively. The conclusions and discussion are presented in Section 6.

\section{DATA AND METHODOLOGIES}

\subsection{Data}

The reanalysis dataset used in this study is provided by Nation Centers for Environmental Prediction (hereafter NCEP) from Nation Centers for Atmospheric Research (NCAR). The daily mean dataset is on a grid with a horizontal resolution of $2.5 \times 2.5$ and 17 vertical pressure levels $(1,000,925,850,700,600,500,400$, $300,250,200,150,100,70,50,30,20$ and $10 \mathrm{hPa})$. The time period from January 1979 to February 2016 of this dataset is analyzed in this study, including geopotential height, air temperature and horizontal wind.

\section{METHODOLOGIES}

\subsection{Objective Blocking Detection Method}

In this study, an objective analysis method of Zhu et al. (2007) to select UB events and we choose the 5 days as the minimum duration of UB events referring to the definition of Davini et al., 2012. The detailed criteria are shown as follows:

(1) There is a closed high-pressure center on $500 \mathrm{hPa}$ over the mid-high latitudes (north of $50^{\circ} \mathrm{N}$ );

(2) The warm high-pressure center lasts for at least 5 days and during this period it is commonly quasi-stationary, sometimes moving eastward or westward with a speed less than 7-8 longitude degree/day; 
(3) Over the blocking region, the westerly jet is significantly decelerated and splits into two branches west of the highpressure center. The two branches, i.e., northern and southern branches, merge in the east of it. The zonal distance between split and merge points is larger than 40-50 longitude degrees.

One of the advantages of this detection method is avoiding the mis-interpretation of the relatively high geopotential height anomalies without anti-cyclonic structure as blocking high (Jin et al., 2009). Though less simple than some other methods, such as T\&M method (Tibaldi and Molteni 1990), this detection method satisfies our need for recognition of the lifetime of blockings, especially for dates of establishment and collapse.

\subsubsection{Momentum and Heat Transport of Transient Eddies}

In this study, we used a decomposition method for the atmospheric variables. According to the principle of physical decomposition (Qian and Jiang 2014), the temporal anomalous fields, such as $u^{\prime}, v^{\prime}$ and $T^{\prime}$, are defined on a calendar date $(\mathrm{t})$ in the year $(\mathrm{y})$ at latitude $(\varphi)$. The detailed method is as follows:

An observational daily mean variable field $\mathrm{F}(\lambda, \varphi, t)_{y}$, such as $\mathrm{u}, \mathrm{v}$ or $\mathrm{T}$, is defined on a calendar date $(\mathrm{t})$ in a year $(\mathrm{y})$ at a spatial grid point of longitude $(\lambda)$ and latitude $(\varphi)$. Then it can be decomposed into a climatological field $\overline{\mathrm{F}}(\lambda, \varphi, t)_{y}$ and temporal anomalous field $\mathrm{F}^{\prime}(\lambda, \varphi, t)_{y}$.

$$
\mathrm{F}(\lambda, \varphi, t)_{y}=\overline{\mathrm{F}}(\lambda, \varphi, t)_{y}+\mathrm{F}^{\prime}(\lambda, \varphi, t)_{y}
$$

The climatological fields are estimated by averaging 38 years (1979-2016) of data based on the NCEP/NCAR reanalysis data on the calendar date $(\mathrm{t})$.

$$
\overline{\mathrm{F}}(\lambda, \varphi, t)_{y}=\sum_{1979}^{2016} \mathrm{~F}(\lambda, \varphi, t)_{y} / 38
$$

The temporal anomalous fields can be derived after removing the climatological fields from the original observations. One is the zonal-averaged anomalous fields on calendar date $(\mathrm{t})$ in year $(\mathrm{y})$ at latitude $(\varphi)$.

$$
[\mathrm{F}(\lambda, \varphi, t)]_{y}^{Z^{\prime}}=\sum_{\lambda=\lambda_{1}}^{\lambda_{2}}\left[\mathrm{~F}(\lambda, \varphi, t)_{y}-\overline{\mathrm{F}}(\lambda, \varphi, t)_{y}\right] /\left(\lambda_{2}-\lambda_{1}+1\right)
$$

This component is referred to as the zonal-averaged (or planetary-scale) anomaly that is used in this paper. The zonalmean momentum and heat transport of transient eddies are represented by $\left[u^{\prime} v^{\prime}\right]$ and $\left[v^{\prime} T^{\prime}\right]$, respectively. The square brackets indicate zonal mean.

\subsubsection{The Generalized E-P Flux}

The generalized Eliassen-Palm flux defined by Andrews and Mcintyre (1976) is used in this study to investigate the wave activity during UB events. According to the transformed Eulerian mean formulation under the approximation of $\beta$ plane, the E-P flux is defined as follows:

$$
\vec{F} \equiv \vec{j} F_{y}+\vec{k} F_{z}
$$

where $F_{y}$ and $F_{z}$ are the meridional and vertical components of EP flux:

$$
\begin{gathered}
F_{y}=-\rho_{0} \overline{u^{\prime} v^{\prime}} \\
F_{z}=\rho_{0} f_{0} R \overline{v^{\prime} T^{\prime}} /\left(N^{2} H\right)
\end{gathered}
$$

where $\rho_{0}$ indicates air density, $u^{\prime}, v^{\prime}$ and $T^{\prime}$ are the disturbance of $u, v$ and $T, f_{0}$ is the Coriolis parameter and $\mathrm{R}$ shows the gas constant for dry air. $\mathrm{N}$ and $\mathrm{H}$ represent buoyancy frequency and scale height, respectively.

Then the divergence of E-P flux is written as:

$$
\nabla \bullet \vec{F}=\frac{\partial F_{y}}{\partial y}+\frac{\partial F_{z}}{\partial z}
$$

\section{THE CLASSIFICATION OF BLOCKING EVENTS AND THE CHARACTERISTIC ANALYSIS OF THE CIRCULATION EVOLUTION}

According to the blocking detection method described in the Method section, 32 winter blocking events over the Ural region are detected during 1979-2015 and the onset and collapse dates of these events are shown in Table 1. Firstly, Figure 1 shows the composited geopotential height and horizontal wind fields on 500 and $850 \mathrm{hPa}$ pressure levels from 5 days prior (Day -5) to 3 days after (Day +3) the onset of UB. On Day -5, the mid-latitudes are dominated by westerly. There are negative geopotential height anomalies west of Ural region, north of Europe, while the Ural region is dominated by weak positive geopotential height anomalies. 2 days after, the negative geopotential height anomalies west of the Ural region are strengthened, forming a weak trough, which is more obvious on $850-\mathrm{hPa}$ pressure level. On Day -1, the positive geopotential height anomalies over the Ural region are significantly strengthened, forming a weak ridge. The trough west of the Ural region is also deepened. In addition, there are negative geopotential height anomalies over the western Siberian Plain, tilting towards northeast-southwest. On Day 0, the Ural positive anomalies are further strengthened, with their center value up to $140 \mathrm{gpm}$, forming an anti-cyclone anomaly on $850-\mathrm{hPa}$ pressure level. The negative Siberian anomalies are also expanded. On Day +3 , the Ural ridge shows a northwestsoutheast tilt. The trough west of the Ural region is significantly weakened, indicating the weakening of the upstream energy and the blocking tends to decay.

Generally, the circulation fields from Day -3 to Day +3 show distinct evolution characteristics, but it is hard to reveal the influences of the troughs and ridges on the lifetime of the blocking high based on the above-mentioned composited results. Therefore, in order to investigate the possible impact of the transient eddies on the lifetime of blocking high, according to the lifetime of the blocking high, the following 
TABLE 1 | Onset and collapse dates of the Ural blocking events during 1979-2015.

\begin{tabular}{|c|c|c|c|c|c|c|c|}
\hline & Onset date & Collapse date & Lasting days (d) & & Onset date & Collapse date & Lasting days (d) \\
\hline 1 & 1979/12/28 & 1980/01/03 & 7 & 17 & 1995/02/15 & $1995 / 02 / 20$ & 6 \\
\hline 2 & 1980/01/27 & 1980/02/05 & 10 & 18 & 2000/01/09 & 2000/01/15 & 7 \\
\hline 3 & 1981/01/17 & 1981/01/22 & 6 & 19 & 2001/02/03 & 2001/02/07 & 5 \\
\hline 4 & 1981/02/20 & $1981 / 02 / 25$ & 6 & 20 & 2003/01/19 & $2003 / 01 / 25$ & 7 \\
\hline 5 & 1982/12/19 & $1982 / 12 / 26$ & 8 & 21 & 2003/02/06 & 2003/02/11 & 6 \\
\hline 6 & 1984/01/13 & 1984/01/18 & 6 & 22 & 2003/12/27 & 2004/01/04 & 9 \\
\hline 7 & 1984/01/23 & 1984/02/03 & 12 & 23 & 2004/01/31 & 2004/02/04 & 5 \\
\hline 8 & 1984/02/07 & 1984/02/12 & 6 & 24 & $2005 / 01 / 23$ & 2005/02/09 & 18 \\
\hline 9 & 1984/02/15 & $1984 / 02 / 23$ & 9 & 25 & 2007/12/08 & 2007/12/12 & 5 \\
\hline 10 & 1984/12/14 & $1984 / 12 / 28$ & 15 & 26 & 2007/12/26 & $2007 / 12 / 31$ & 6 \\
\hline 11 & 1985/01/05 & 1985/01/12 & 8 & 27 & 2009/02/15 & $2009 / 02 / 23$ & 9 \\
\hline 12 & 1985/02/20 & 1985/02/28 & 9 & 28 & 2010/02/02 & 2010/02/06 & 5 \\
\hline 13 & 1986/01/28 & 1986/02/03 & 7 & 29 & 2011/01/07 & 2011/01/11 & 5 \\
\hline 14 & 1988/01/22 & 1988/01/31 & 10 & 30 & 2012/01/17 & 2012/01/29 & 13 \\
\hline 15 & 1990/02/15 & $1990 / 02 / 21$ & 7 & 31 & 2012/12/10 & 2012/12/19 & 10 \\
\hline 16 & 1995/01/17 & 1995/01/22 & 6 & 32 & 2016/02/10 & $2016 / 02 / 14$ & 5 \\
\hline
\end{tabular}

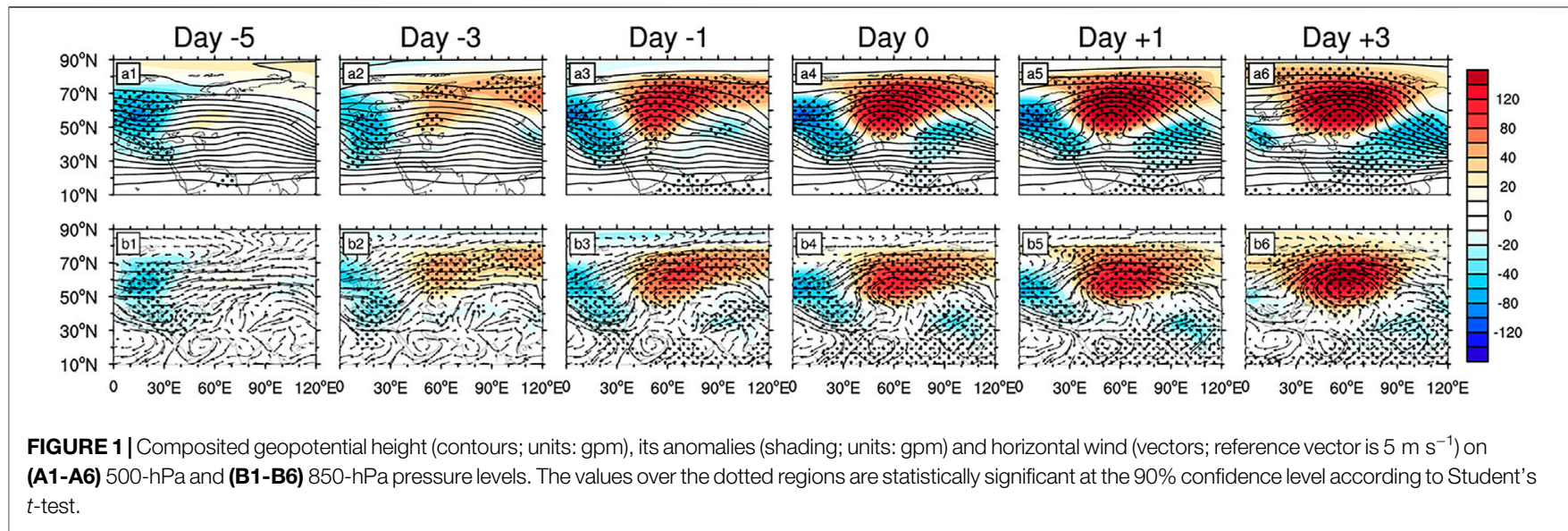

parts classify these blocking events into three types of events: short (5-6 days), medium (7-9 days) and long ( $\geq 10$ days) lifetime events. These events are sorted by their onset dates. $3,4,6,8,16,17,19,21,23,25,26,28,29$ and 32 are sorted as short events, $1,5,9,11,12,13,15,18,20,22$ and 27 as medium events and 2, 7, 10, 14, 24, 30 and 31 as long events. The Ural region in this study is outlined as the region between $40^{\circ} \mathrm{E}-80^{\circ} \mathrm{E}$. In the following parts, the characteristics of the transient eddies during different lifetime blocking events are analyzed according to the composited results of transient eddy momentum flux and heat transport.

\section{CHARACTERISTIC ANALYSIS OF TRANSIENT EDDY MOMENTUM TRANSPORT}

The onset of blocking high is often accompanied by the abrupt collapse of westerly. The westerly jet could split into the northern and southern branches. Strong transient transport is associated with strong jet stream (Hoskin and Ambrizzi 1993; Ren et al.,
2011). Therefore, the characteristics of upper-level jet stream during the blocking events are also analyzed, with positive $\left[u^{\prime} v^{\prime}\right]$ denoting the northward eddy momentum flux.

Firstly, the westerly jet stream and transient eddy momentum transport from Day -3 to Day +3 of the short UB events are shown in Figure 2 (a1-a7). From Figure 2, it can be found that during the blocking events westerly splits into two branches, i.e., sub-tropic and polar jets, south and north of the Ural blocking, respectively. Before the onset of $U B$, both the two jet streams are relatively weak, with the positive $\left[u^{\prime} v^{\prime}\right]$ dominating the mid-upper troposphere between $40^{\circ} \mathrm{N}-60^{\circ} \mathrm{N}$ (Figures 3a1,a2), indicating the poleward transport of eddy momentum flux by transient eddies. On Day -3 , the positive $\left[u^{\prime} v^{\prime}\right]$, up to $70 \mathrm{~m}^{2} \mathrm{~s}^{-2}$, centers around $65^{\circ} \mathrm{N}, 300 \mathrm{hPa}$, while there is weak negative $\left[u^{\prime} v^{\prime}\right]$ north of $80^{\circ} \mathrm{N}$, forming the convergence of eddy momentum flux around $80^{\circ} \mathrm{N}$. Two days after, the westerly jet stream splits up and the polar jet is shifted northward to $70^{\circ} \mathrm{N}$, forming the strong westerly belt between $50^{\circ} \mathrm{N}-70^{\circ} \mathrm{N}$. The positive center of $\left[u^{\prime} v^{\prime}\right]$ is strengthened and the convergence center is shifted equatorward to $75^{\circ} \mathrm{N}$. The convergence of eddy momentum flux and the decelerated 

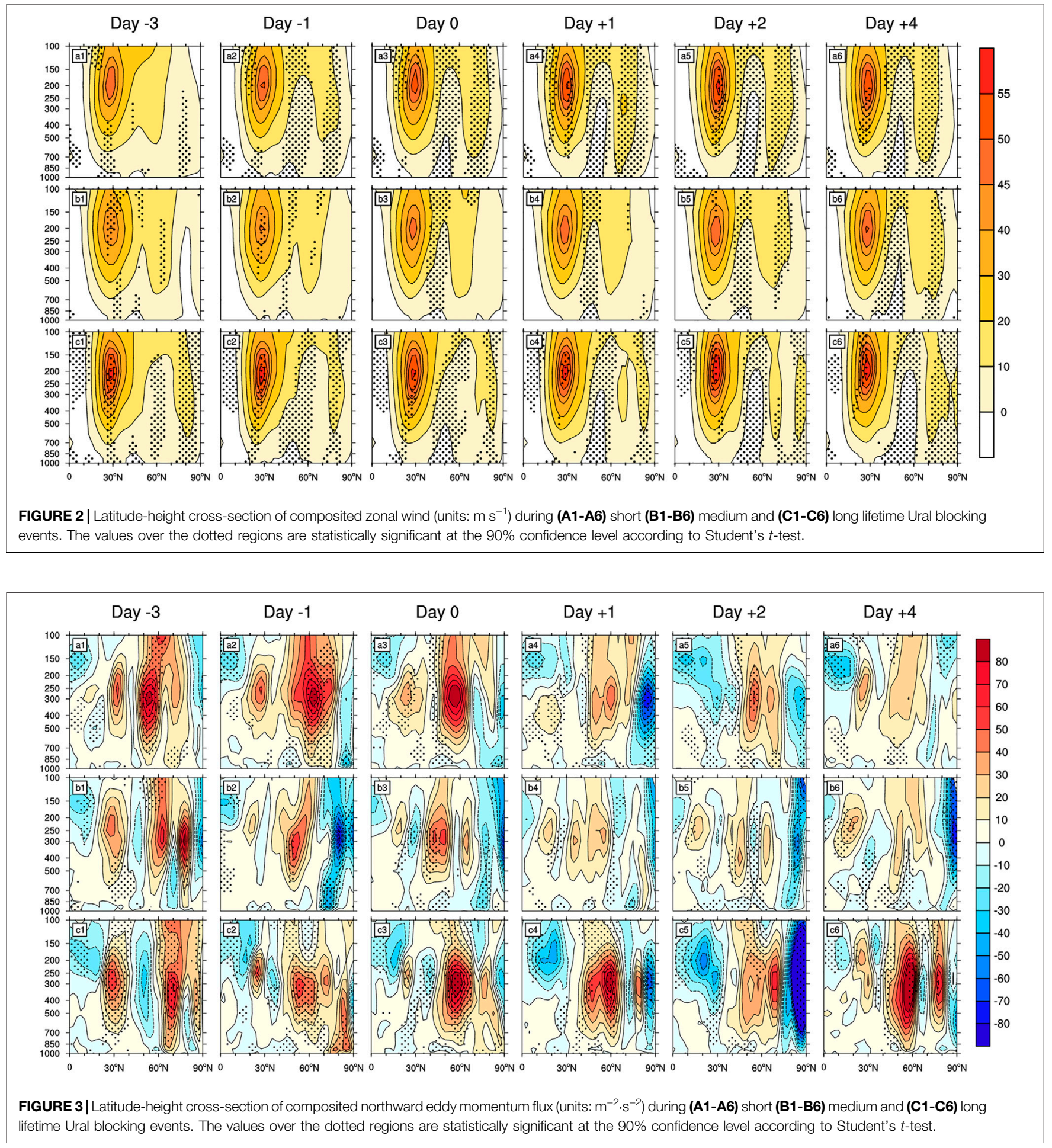

westerly are favorable for the establishment of the blocking high (Xu and Jin 2011; Li et al., 2019). On Day 0, the sub-tropic jet stream is accelerated, with its maximum up to $55 \mathrm{~m} \mathrm{~s}^{-1}$ around $30^{\circ} \mathrm{N}, 200 \mathrm{hPa}$. The convergence center of eddy momentum flux is shifted to $70^{\circ} \mathrm{N}$, maintaining the UB. One day after, the two jet streams are continuously strengthened, reaching their maximum. The negative $\left[u^{\prime} v^{\prime}\right]$ also reaches its maximum and then $\left[u^{\prime} v^{\prime}\right]$ is weakened, along with the weakening and poleward shift of eddy momentum convergence. On Day +4 , both the two jet streams are significantly decelerated. The transport of eddy momentum grows weaker over the blocking region and the blocking high tends to collapse.

Then, the westerly jet stream and transient eddy momentum transport from Day -3 to Day +3 of the medium lifetime UB 
events are also shown Figure 2 (b1-b7). Similar to the short events, the westerly in the medium events also splits into the northern and southern branches. On Day -3, the sub-tropic jet is located around $30^{\circ} \mathrm{N}, 200 \mathrm{hPa}$, up to $45 \mathrm{~m} \mathrm{~s}^{-1}$, while the polar jet is located between $50^{\circ} \mathrm{N}-70^{\circ} \mathrm{N}$. [ [ $u^{\prime} v^{\prime}$ ] is positive (negative) south (north) of $65^{\circ} \mathrm{N}$ (Figure 3b1). Then [ $\left.u^{\prime} v^{\prime}\right]$ is continuously strengthened. On Day -1 , the positive [ $\left.u^{\prime} v^{\prime}\right]$ centers around $50^{\circ} \mathrm{N}, 400 \mathrm{hPa}$, up to $70 \mathrm{~m}^{2} \mathrm{~s}^{-2}$, while the negative [ $u^{\prime} v^{\prime}$ ] dominates the whole troposphere over the high latitudes, with the convergence centering around $60^{\circ} \mathrm{N}$. On Day 0 , both the two jet streams are enhanced, with the polar jet core located over the upper troposphere at $60^{\circ} \mathrm{N}$. The transient eddy momentum transport is slightly weakened, but still converges around $60^{\circ} \mathrm{N}$, favorable for the maintenance of UB. After that, the sub-tropic jet stream is continuously accelerated, while the polar jet is strengthened and shifted northward. On Day $+2\left[u^{\prime} v^{\prime}\right]$ over the high latitudes is greatly reduced, with its center descending to $500 \mathrm{hPa}$. The convergence center of eddy momentum fluxes is also weakened and shifted southward to around $50^{\circ} \mathrm{N}$. The northward eddy momentum flux over the middle latitudes is also greatly weakened, with its center descending to $500 \mathrm{hPa}$. The convergence center at $70^{\circ} \mathrm{N}$ appears again and is strengthened 2 days after.

The results of the long lifetime UB events are also shown in Figure 2 (c1-c7). The westerly still splits into two branches and the sub-tropic jet is stronger, which is up to $55 \mathrm{~m} \mathrm{~s}^{-1}$. Polar jet stream is located north of $75^{\circ} \mathrm{N}$, indicating the strong development of the Ural ridge. On Day -3, the westerly already splits up. The northward transport of eddy momentum converges in the lower troposphere around $75^{\circ} \mathrm{N}$. Then, the negative [ $\left.u^{\prime} v^{\prime}\right]$ is weakened. On Day -1 , the westerly jet between $55^{\circ} \mathrm{N}-75^{\circ} \mathrm{N}$ is decelerated and the transient eddies around $55^{\circ} \mathrm{N}$ transport energy for the blocking high. After the onset of the blocking high, the westerly over the blocking region is continuously weakened and the sub-tropic jet is significantly strengthened. On Day +1 , the positive [ $\left.u^{\prime} v^{\prime}\right]$ at $55^{\circ} \mathrm{N}$ is up to $80 \mathrm{~m}^{2} \mathrm{~s}^{-2}$. The eddy momentum fluxes converge around $65^{\circ} \mathrm{N}$ and are strengthened continuously, weakening after Day +7 (not shown), which is favorable for the long-time maintenance of the blocking high.

\section{CHARACTERISTIC ANALYSIS OF TRANSIENT EDDY HEAT TRANSPORT}

The synoptic-scale transient eddies are closely linked to the growth of atmospheric baroclinicity in the lower troposphere. The transient eddies are due to the instability of temporal mean flow, which is mainly produced in the strong baroclinic region and moves towards the weak baroclinic region. The outlet of the jet stream (weak baroclinicity) is where the blocking high happens (Diao et al., 2004). Transient eddies usually propagate along the gradient and play an important role in the development of the mid-latitude baroclinic systems and establishment of blocking high. Similar to the eddy momentum transport, the positive/negative $\left[v^{\prime} T^{\prime}\right]$ denotes the poleward/equatorward transport of eddy heat flux.
Figure 4 (a1-a7) and Figure 5 (a1-a7) shows the latitudeheight cross-section of the temperature disturbance and [ $\left.v^{\prime} T^{\prime}\right]$ during the short lifetime UB events. During the short UB events, the temperature disturbance anomalies are mainly distributed over the middle and high latitudes and there is an "upper cold, lower warm" pattern vertically over the high latitudes, with the transition layer around $300 \mathrm{hPa}$. The low latitudes are dominated by relatively weak warm anomalies. On Day -3 , significant negative temperature anomalies, upper to $-4 \mathrm{~K}$, appear in the upper troposphere over the high latitudes, while the positive anomalies mainly exist in the lower troposphere between $1,000-700 \mathrm{hPa}$. There are northward eddy heat fluxes transported by transient eddies in the lower troposphere over the mid-high latitudes, whereas in the upper troposphere between $70^{\circ} \mathrm{N}-80^{\circ} \mathrm{N}$ exists weak southward transport of eddy heat flux. 2 days after, the near-surface positive temperature anomalies are enhanced and extend upward to $400 \mathrm{hPa}$. Then, the temperature anomalies in the upper troposphere are reduced drastically, leading to the weakening of the vertical temperature gradient over the blocking region and growth of atmospheric baroclinicity (Hu et al., 2018). Consequently, the transient eddies transport eddy heat flux along the temperature gradient. [ $\left.v^{\prime} T^{\prime}\right]$ is significantly strengthened and there is weak convergence of eddy heat flux on $300 \mathrm{hPa}$ around $50^{\circ} \mathrm{N}$. The transient eddies in the lower troposphere at $50^{\circ} \mathrm{N}$ transport eddy heat fluxes northward and provide energy for the establishment of the blocking high. On Day 0, there is northward transport of eddy heat flux over the blocking region and the convergence region is located in the upper troposphere around $70^{\circ} \mathrm{N}$, transporting heat fluxes to the lower troposphere. The positive temperature anomalies over the high latitudes reach the maximum 1 day after, when the atmospheric baroclinicity also reaches its maximum. There is northward eddy heat flux transport throughout the whole troposphere and the heat transport in the convergence zone is favorable for the establishment of blocking high. On Day $+4\left[T^{\prime}\right]$ throughout troposphere are weakened a lot and the southward transport of eddy heat flux by the transient eddies is reduced and diminished, leading to the weakening and collapse of blocking high.

The transient eddy heat flux transport during medium lifetime UB events is shown in Figure 5 (b1-b7). During these events, positive [ $T$ '] appears in the lower latitudes and shows little changes, which has negligible impact on the blocking events and therefore will not be discussed in the following parts of this study. There still exists an "upper cold, lower warm" vertical pattern over the mid-high latitudes. However, the positive [ $\left.T^{\prime}\right]$ is relatively weak and the negative $\left[T^{\prime}\right]$ in the upper troposphere also appears very late. On Day -3 , the positive center of [ $\left.T^{\prime}\right]$ is located south of $60^{\circ} \mathrm{N}$ in the lower troposphere and is also relatively weak. There is large-scale transient eddy heat transport over the mid-high latitudes. There is northward/ southward transport of eddy heat flux south/north of $60^{\circ} \mathrm{N}$ in the mid-lower troposphere, indicating the convergence of eddy heat fluxes. 2 days after, the lower-level positive $\left[T^{\prime}\right]$ is strengthened and the atmospheric baroclinicity gradually grows stronger. The convergence center is also shifted northward to $60^{\circ} \mathrm{N}$ and negative $\left[T^{\prime}\right]$ in the lower troposphere 

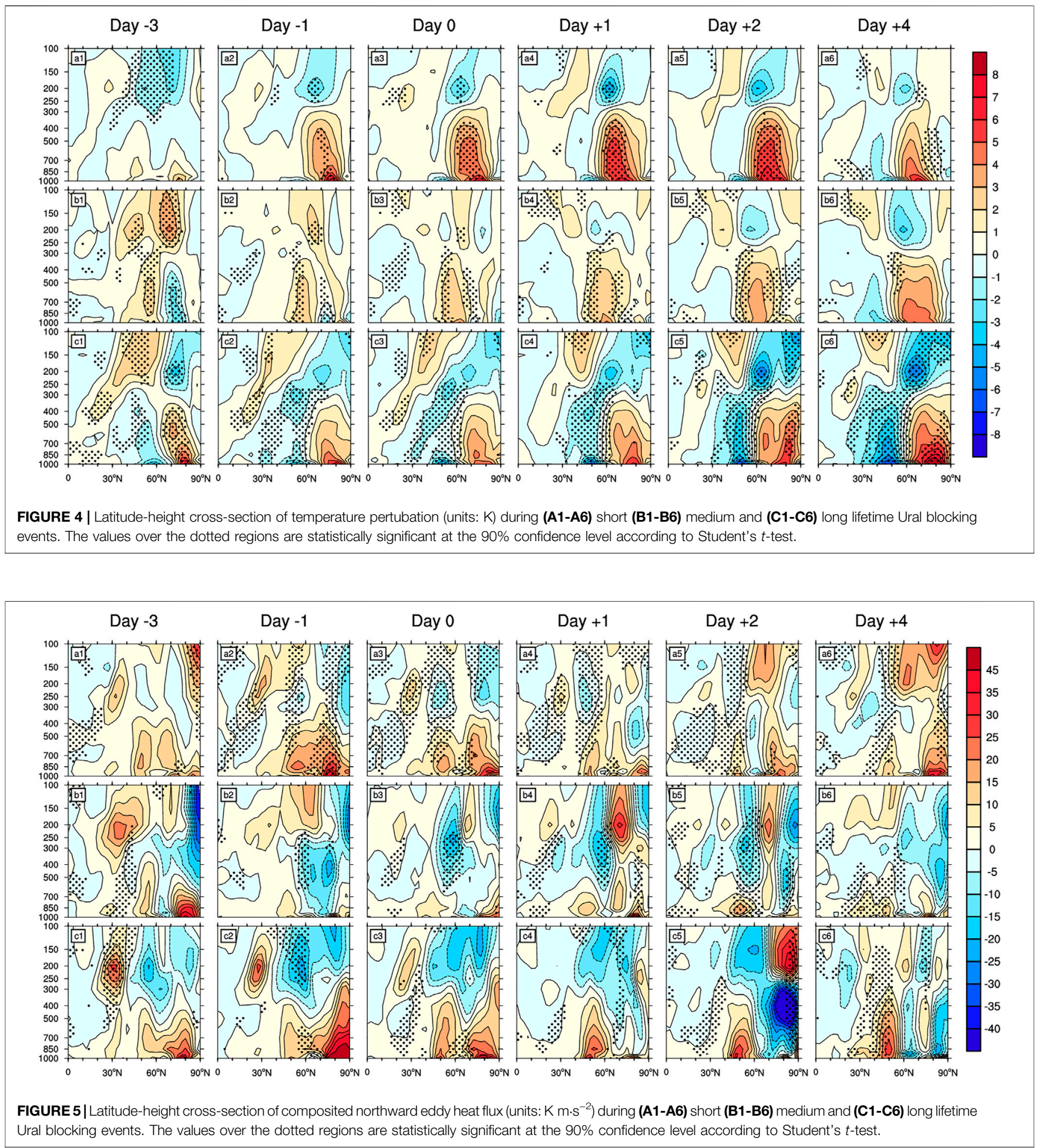

reaches its maximum, with its center descending to $500 \mathrm{hPa}$. The strong heat transport over the convergence zone provides energy for the establishment of blocking high. After the onset of the blocking events, the "upper cold, lower warm" pattern over the high latitudes is more significant and the extent and intensity of $\left[T^{\prime}\right]$ are also strengthened, weakening the temperature gradient in the lower level. According to the thermal wind balance, the thermal wind is weakened and the mid-latitude westerly is decelerated, which is favorable for the enhancement of heat transport and establishment of blocking high. On Day +4 $\left[v^{\prime} T^{\prime}\right]$ in the mid-lower troposphere is weakened, leading to the decay and collapse of the blocking high. 

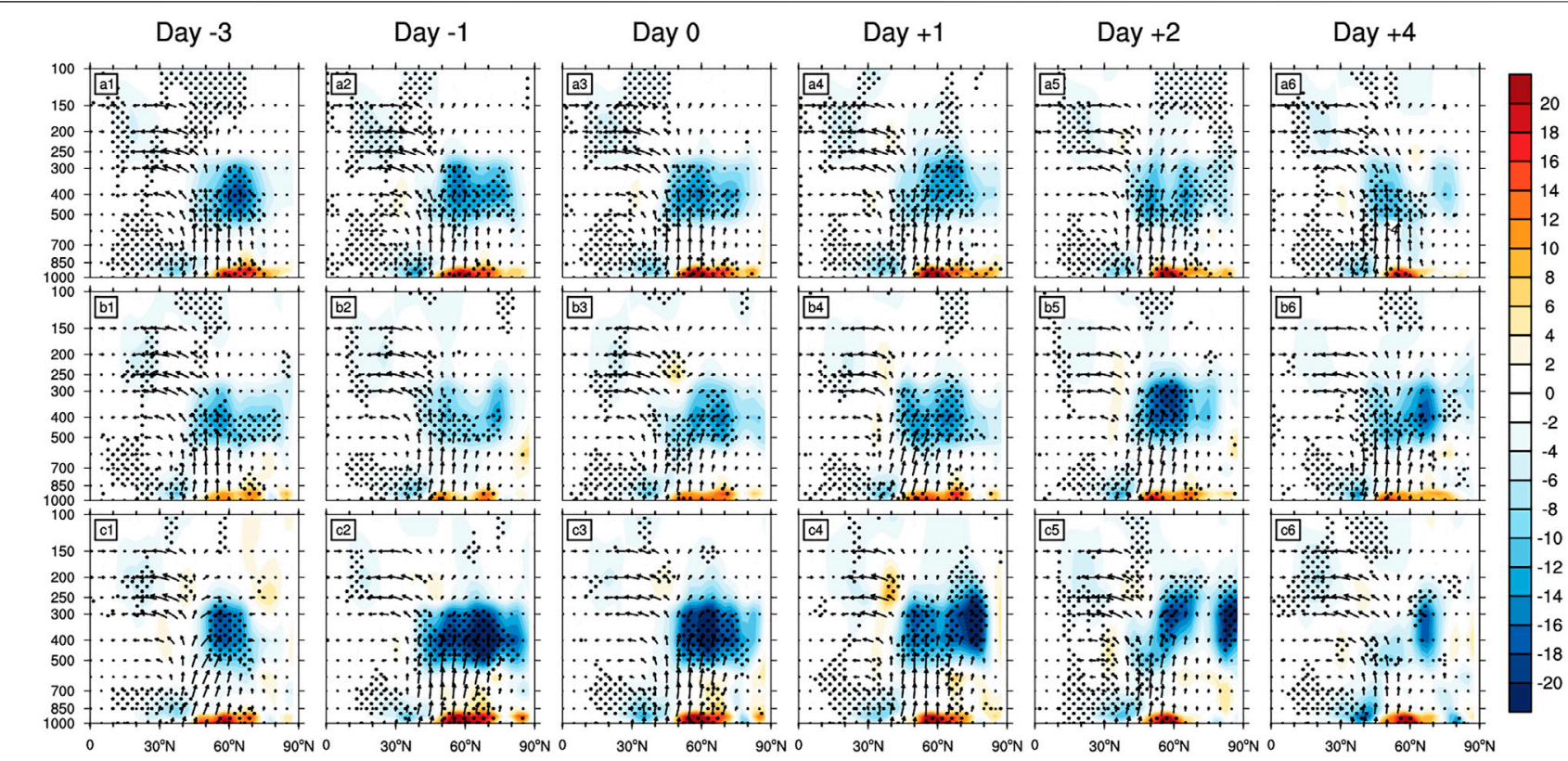

FIGURE 6 | Latitude-height cross-section of composited E-P flux (vectors; scaled by square root of air pressure; the vertical and meridional components are scaled by $10^{4}$ and $10^{6}$, respectively; units: $\mathrm{m} \cdot \mathrm{s}^{-2}$; the reference vector denotes $10 \mathrm{~m} \mathrm{~s}^{-2}$ ) and its divergence (shading; units: $\mathrm{s}^{-2}$ ) during (A1-A6) short (B1-B6) medium and (C1-C6) long lifetime Ural blocking events. The values over the dotted regions are statistically significant at the 90\% confidence level according to Student's t-test.

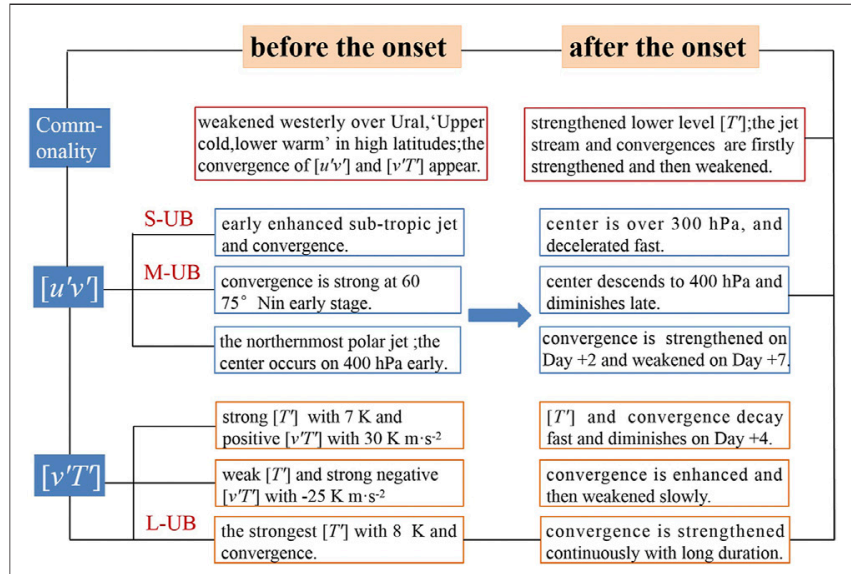

FIGURE 7 | Comparison results of transient eddy characteristics among different lifetime UB events. SUB, MUB and LUB denote short, medium and long lifetime UB events.

Figure $4(c 1-c 7)$ and Figure 5 (c1-c7) show the latitude-height cross-section of the temperature disturbance and the transient eddy heat flux during the long lifetime UB events. Different from the short and medium blocking events, [ $\left.T^{\prime}\right]$ is significant throughout the whole life cycle of the blocking events. There is an "upper warm, lower cold" pattern over the middle latitudes, contrast to that over the high latitudes. The negative [ $\left.T^{\prime}\right]$ throughout the whole troposphere implies that the highpressure ridge during the blocking events induces the southward intrusion of polar cold air mass and strengthens the surface cold high pressure center, which is favorable for cold extremes. On Day -3, the eddy heat flux converges around $60^{\circ} \mathrm{N}$ in the mid-lower troposphere. After that [T'] over the high latitudes is further enhanced and the meridional heat transport splits on $400 \mathrm{hPa}$, with southward transport in the upper troposphere and northward transport in the lower troposphere, favorable for the establishment of blocking high. After the onset of the blocking events, the lower-tropospheric [ $\left.T^{\prime}\right]$ is significantly enhanced. According to the thermal wind balance, the mid-latitude westerly is decelerated. On Day +2 , the transient eddy heat transport in the convergence zone around $65^{\circ} \mathrm{N}$ is further enhanced, with negative $\left[v^{\prime} T^{\prime}\right]$ up to $50 \mathrm{~K} \mathrm{~m} \cdot \mathrm{s}^{-2}$, favorable for the long-time maintenance of blocking high.

\section{CONCLUSION AND DISCUSSION}

In this study, the wintertime UB events are selected according to objective analysis method and these events are further classified into three types according to their lifetime, i.e., short, medium and long lifetime UB events. Based on the analysis of the characteristics of transient eddy momentum and heat transport during UB events with different lifetime, we find the convergence of transient eddy flux plays an important role in the lifetime of UB and the transient eddy shows different characteristics during UB events with different lifetimes. As an important diagnostic, the E-P flux, which takes into account thermal and momentum transport by transient eddies together, can be applied in this study to diagnose the wave activities associated with blocking high and verify the above conclusions.

This study further analyzes the E-P flux and its divergence during the UB events, which are shown in Figure 6. The E-P flux 
divergence has an important influence on the evolution of basic zonal wind in the upper-level jet stream region, namely wavemean flow interaction. The acceleration and deceleration of basic zonal flow mainly result from the meridional transportation of eddy momentum flux (Zhang and Ni 1991). It can be found from Figure 6 that there is obvious E-P flux convergence in the middle troposphere between $50^{\circ} \mathrm{N}-70^{\circ} \mathrm{N}$ during all the three types of UB, consistent with the above-mentioned results. The westerly could be decelerated, or even turn into easterly, where the E-P flux converges. It can be noted from Figure 2 that the westerly over the blocking region is significantly decelerated, providing favorable background for the establishment of UB. On Day -3, there already exists obvious E-P flux divergence in the middle troposphere over the blocking region during all these blocking events. However, the convergence in the medium UB events is relatively weak, while the convergence in the long UB events is the strongest. Then, the convergence grows stronger gradually and the westerly over the blocking region is decelerated, favorable for the establishment of the blocking high. The convergence of the E-P flux after the onset of the blocking events is gradually weakened during the short UB events, with only $-8 \mathrm{~s}^{-2}$ on Day +4 , which could be one of the possible causes of its short life cycle. The E-P flux convergence during the medium UB events is significantly strengthened and can still maintain its strength 2 days after, favorable for the maintenance of blocking high. On Day +1 of the long UB events, the E-P flux convergence reaches $-20 \mathrm{~s}^{-2}$, with a northward shifted center and larger extent, indicating the strong development of the Ural ridge. Consistent with the results of transient eddy momentum and heat fluxes, the strong E-P flux divergence persists throughout the whole long blocking events, only showing a weakening trend on Day +4 , favorable for the long duration of blocking high.

In general, the E-P flux and transient eddy momentum and heat transport show similar characteristics. Before the onset of $\mathrm{UB}$, westerly splits into two branches, and the lower-level [ $\left.T^{\prime}\right]$ is strengthened in the middle and high latitudes, leading to a weakened westerly over Ural. Meanwhile, the strong convergence of transient eddies and E-P fluxes occur over the Ural region. All of these are favorable for the establishment of UB. After the onset of UB, both two jet streams and the convergence of the transient eddy momentum and heat transport are significantly enhanced, leading to the long-term maintenance of UB. Therefore, our results are consistent with some previous studies, i.e., the weakened westerly and the enhanced convergence of transient eddy are favorable for the establishment of UB (e.g., $\mathrm{Xu}$ and Jin 2011; Li et al., 2019).

Further we find the transient eddy shows different characteristics during UB events with different lifetimes. The comparison results are shown in Figure 7. Some studies found that the strong baroclinicity of the atmosphere and the enhanced split-jet flow are favorable for the establishment and long maintenance of UB (Luo 2005; Luo and Zhang 2020b). Consistent with these studies, we found that the jet stream, the [T'] and the baroclinicity of the atmosphere during long events are the strongest among all the UB events. Also, we found that the convergence of transient eddies is the strongest during the long UB events. The convergence of transient eddy momentum and heat flux appears early with a strong intensity, further strengthened on Day +2 and weakened on Day +7 , favorable for the long-time maintenance of UB. The jet stream and $\left[T^{\prime}\right]$ during short UB events are strengthened on Day -1, with a strong convergence of eddy momentum flux, which also decay fast, leading to an early collapse of UB. The convergence of eddy momentum and heat flux during medium UB events appears on Day -3 and further strengthens after the onset of UB. Though weaker than those during long events, the transient eddies can still maintain UB for a long time. In addition, consistent with the result of Kong and $\mathrm{Hu}$ (2014) that the strong upward propagation of wave fluxes over the Ural region favors the long-time maintenance and development of UB events, the upward propagating wave activity over Ural during long UB events is also the strongest and most long-lasting in this study.

This study investigated the characteristics of transient eddies during the evolution of wintertime UB events, which is consistent with Athar and Lupo (2010) and Xu and Jin (2011). We also find that strong baroclinicity and strong convergence of transient eddies with long duration are closely linked to the long-time maintenance of UB. However, in this study only the characteristics of transient eddies are investigated around the onset of UB, lacking the discussion on the results during the other stages of UB (growth, mature, and decay). In addition, considering that blocking high is a result of interaction between different largescale waves (Austin 1980), the possible role of stationary momentum and heat fluxes should also be investigated. Therefore, the dynamical mechanism of the momentum and heat exchange between mean and eddy flow during the blocking events will be further investigated in the future study. Also, different blocking regions will be studied and model results will be used to validate the findings from observation.

\section{DATA AVAILABILITY STATEMENT}

Publicly available datasets were analyzed in this study. This data can be found here: https://psl.noaa.gov/data/gridded/data.ncep. reanalysis.html

\section{AUTHOR CONTRIBUTIONS}

JZ contributed to manuscript original draft. YL provided the conceptualization and manuscript review and editing. ZW and $\mathrm{YZ}$ provided the formal analysis, validation and funding acquisition. YL provided the data curation

\section{FUNDING}

This research was supported by the National Key Research \& Development (R\&D) Program of China (2019YFA0606801), the Natural Science Foundation of China (41975111), the Natural Science Foundation of Gansu Province (20JR5RA120) and Research Project of Lanzhou Institute of Arid Meteorology, China Meteorological Administration (KYS2021SSKY01). 


\section{REFERENCES}

Andrews, D. G., and Mcintyre, M. F. (1976). Planetary Waves in Horizontal and Vertical Shear: Asymptotic Theory for Equatorial Waves in Weak Shear. J. Atmos. Sci. 33, 2049-2053. doi:10.1175/1520-0469(1976)033<2049: pwihav $>2.0 . \mathrm{co} ; 2$

Athar, H., and Lupo, A. R. (2010). Scale Analysis of Blocking Events from 2002 to 2004: a Case Study of an Unusually Persistent Blocking Event Leading to a Heat Wave in the Gulf of Alaska during August 2004. Adv. Meteorol. 12, 185-194. doi:10.1155/2010/610263

Austin, J. F. (1980). The Blocking of Middle Latitude westerly Winds by Planetary Waves. Q.J R. Met. Soc. 106, 327-350. doi:10.1002/qj. 49710644807

Bueh, C., Fu, X., and Xie, Z. W. (2011). Large-scale Circulation Features Typical of Wintertime Extensive and Persistent Low Temperature Events in china. Atmos. Oceanic Sci. Lett. 4, 7. doi:10.1080/16742834

Charney, J. G., and Devore, J. G. (1979). Multiple Flow Equilibria in the Atmosphere and Blocking. J. Atmos. Sci. 36, 1205-1216. doi:10.1175/15200469(1979)036<1205:mfeita>2.0.co;2

Davini, P., Cagnazzo, C., Gualdi, S., and Navarra, A. (2012). Bidimensional Diagnostics, Variability, and Trends of Northern Hemisphere Blocking. J. Clim. 25, 6496-6509. doi:10.1175/JCLI-D-12-00032.1

Diao, Y., Li, J., and Luo, D. (2004). A Dynamic Study of the Interaction between Transient Eddies and Blocking. Chin. J. Atmos. Sci. (in Chinese). 28, 24.

Diao, Y., Li, J., and Luo, D. (2006). A New Blocking index and its Application: Blocking Action in the Northern Hemisphere. J. Clim. 19, 4819-4839. doi:10. 1175/jcli3886.1

Ding, Y., and Johnny, C. L. (2005). The East Asian Summer Monsoon: an Overview. Meteorol. Atmos. Phys. 89, 117-142. doi:10.1007/s00703-0050125-z

Feng, J., Li, J., Liao, H., and Zhu, J. (2019). Simulated Coordinated Impacts of the Previous Autumn North Atlantic Oscillation (NAO) and winter El Niño on winter Aerosol Concentrations over Eastern China. Atmos. Chem. Phys. 19, 10787-10800. doi:10.5194/acp-19-10787-2019

Hoskins, B. J., and Ambrizzi, T. (1993). Rossby Wave Propagation on a Realistic Longitudinally Varying Flow. J. Atmos. Sci. 50, 1661-1671. doi:10.1175/15200469(1993)050<1661:rwpoar >2.0.co;2

$\mathrm{Hu}$, S., Cheng, J., Xu, M., and Chou, J. (2018). Three-pattern Decomposition of Global Atmospheric Circulation: Part II-Dynamical Equations of Horizontal, Meridional and Zonal Circulations. Clim. Dyn. 50, 2673-2686. doi:10.1007/ s00382-017-3763-1

Huang, R. H., and Zou, H. (1989). Interaction between Upward Propagating Planetary Waves and Zonal Mean Flow in a Spherical Baroclinic Atmosphere. Chin. J. Atmos. Sci. (in Chinese) 392, 1006-9895. doi:10. 3878/j.issn

Jin, R. H., Li, Y., and Wang, S., 2009. Comparison and Analysis Among Four Objective and Quantificational Blocking Indexes. Plateau Meteorology.28, 5 .

Kong, W., and Hu, Y. (2014). Influence of Stratospheric Nam Anomalies on the Ural Blocking High. Acta Scientiarum Naturalium Universitatis Pekinensis. (in Chinese) 50, 445-455. doi:10.13209/j.0479-8023.2014.082

Li, Y., Lu, Y., and Wang, C. (2020). Characteristics of thermal and Momentum Transport during the Lifetime of Ural Blocking Highs. Int. J. Climatol. 40, 77-93. doi:10.1002/joc.6195

Li, Y., Zhang, J., Lu, Y., Zhu, J., and Feng, J. (2019). Characteristics of Transient Eddy Fluxes during Blocking Highs Associated with Two Cold Events in China. Atmosphere 10, 235. doi:10.3390/atmos10050235

Luo, D. (2003). A Barotropic Envelope Rossby Soliton Model for Block Eddy Interaction. Part I: Effect of Topography. J. Atmos. Sci. 62, 3839-3859. doi:10. 1175/JAS3573.1

Luo, D. (2005). Why Is the north atlantic Block More Frequent and Long-Lived during the Negative NAO Phase. Geophys. Res. Lett. 32, 20804. doi:10.1029/ 2005GL022927

Luo, D., Cha, J., Zhong, L., and Dai, A. (2014). A Nonlinear Multiscale Interaction Model for Atmospheric Blocking: The Eddy-Blocking Matching Mechanism. Q.J.R. Meteorol. Soc. 140, 1785-1808. doi:10.1002/qj.2337
Luo, D., and Chen, Z. (2005). The Role of Land-Sea Topography in Blocking Formation in a Block-Eddy Interaction Model. J. Atmos. Sci. 63, 3056-3065. doi:10.1175/JAS3774.1

Luo, D., Xiao, Y., Diao, Y., Dai, A., Franzke, C. L. E., and Simmonds, I. (2016b). Impact of Ural Blocking on winter Warm Arctic-Cold Eurasian Anomalies. Part II: the Link to the north atlantic Oscillation. J. Clim. 29, 3949-3971. doi:10. 1175/jcli-d-15-0612.1

Luo, D., Xiao, Y., Yao, Y., Dai, A., Simmonds, I., and Franzke, C. L. E. (2016a). Impact of Ural Blocking on winter Warm Arctic-Cold Eurasian Anomalies. Part I: Blocking-Induced Amplification. J. Clim. 29, 3925-3947. doi:10.1175/ JCLI-D-15-0611.1

Luo, D., and Zhang, W. (2020b). A Nonlinear Multiscale Theory of Atmospheric Blocking: Dynamical and Thermodynamic Effects of Meridional Potential Vorticity Gradient. J. Atmos. Sci. 77. doi:10.1175/JAS-D-20-0004.1

Luo, D., and Zhang, W. (2020). A Nonlinear Multiscale Theory of Atmospheric Blocking: Eastward and Upward Propagation and Energy Dispersion of Tropospheric Blocking Wave Packets. J. Atmos. Sci. 77, 4025-4049. doi:10. 1175/JAS-D-20-0153.1

Luo, D., Zhang, W., Zhong, L., and Dai, A. (2019). A Nonlinear Theory of Atmospheric Blocking: A Potential Vorticity Gradient View. J. Atmos. Sci. 76, 2399-2427. doi:10.1175/jas-d-18-0324.1

Lupo, A. R., and Smith, P. J. (1995). Climatological Features of Blocking Anticyclones in the Northern Hemisphere. Tellus A. 47, 439-456. doi:10. 1034/j.1600-0870.1995.t01-3-00004.x

Ma, J., and San Liang, X. (2017). Multiscale Dynamical Processes Underlying the Wintertime atlantic Blockings. J. Atmos. Sci. 74, 3815-3831. doi:10.1175/jas-d16-0295.1

Masato, G., Hoskins, B. J., and Woollings, T. (2013). Wave-breaking Characteristics of Northern Hemisphere winter Blocking: A TwoDimensional Approach. J. Clim. 26, 4535-4549. doi:10.1175/jcli-d-12-00240.1

Mokhov, I. I., Akperov, M. G., Prokofyeva, M. A., Timazhev, A. V., Lupo, A. R., and Le TreutLe, H. (2013). Blockings in the Northern Hemisphere and Euro-atlantic Region: Estimates of Changes from Reanalysis Data and Model Simulations. Dokl. Earth Sc. 449, 430-433. doi:10.1134/s1028334x13040144

Nakamura, H., Nakamura, M., and Anderson, J. L. (1997). The Role of High- and Low-Frequency Dynamics in Blocking Formation. Mon. Wea. Rev. 125, 2074-2093. doi:10.1175/1520-0493(1997)125<2074:trohal >2.0.co;2

Qian, W., and Jiang, M. (2014). Early Signals of Synoptic-Scale Atmospheric Anomalies Associated with the Summer Low Temperature Events in Northeast china. Meteorol. Atmos. Phys. 124, 33-46. doi:10.1007/s00703013-0306-0

Ren, X., Yang, X., Zhou, T., and Fang, J. (2011). Diagnostic Comparison of Wintertime East Asian Subtropical Jet and Polar-Front Jet: Large-Scale Characteristics and Transient Eddy Activities. Acta Meteorol. Sin. 25, 21-33. doi:10.1007/s13351-011-0002-2

Shi, N., and Nakamura, H. (2020). A New Detection Scheme of Wave-Breaking Events with Blocking Flow Configurations. J. Clim. 34, 1-55. doi:10.1175/JCLID-20-0037.1

Shi, N., and Wang, Y. (2021). SuolangtajieEnergetics of Boreal Wintertime Blocking Highs Around Ural Mountains. J. Meteorol. Res. 36, 1-21. doi:10. 1007/s13351-022-1069-7

Takaya, K., and Nakamura, H. (2005). Mechanisms of Intraseasonal Amplification of the Cold Siberian High. J. Atmos. 62, 4423-4440. doi:10.1175/jas3629.1

Tibaldi, S., and Molteni, F. (1990). On the Operational Predictability of Blocking. Tellus A 42, 343-365. doi:10.1034/j.1600-0870.1990.t01-2-00003.x

Wan, H., and Luo, D. (2009). The Relationship between Wintertime Blockings in the north Hemisphere and north atlantic Oscillation. J. Trop. Meteorol. (in Chinese) 25, 61560084-61566204. doi:10.1016/S1003-6326(09)

$\mathrm{Xu}, \mathrm{H}$., and Jin, R. H. (2011). Analysis on the Effect of the Transient Eddy on Ural Blocking High in 2008. Plateau. Meteorol. (in Chinese) 45, 286-298. doi:10. 1111/j.1469-8986.2007.00615.x

Yao, Y., Luo, D., Dai, A., and Simmonds, I. (2017). Increased Quasi Stationarity and Persistence of winter Ural Blocking and Eurasian Extreme Cold Events in Response to Arctic Warming. Part I: Insights from Observational Analyses. J. Clim. 30, 3549-3568. doi:10.1175/jcli-d-16-0261.1

Ye, P., Li, Y., Wang, S., Wang, X., and Shang, K. (2015). Variation Characteristics of Different Atmospheric Blocking and its Influence on Temperature over 
Northern Hemisphere. J. Lanzhou Univ. (Natural Sciences) (in Chinese) 51, 639-645. doi:10.13885/j.issn.0455-2059.2015.05.008

Yeh, C. T. (2010). On Energy Dispersion in the Atmosphere. J. Atmos. Sci. 6, 1-16. doi:10.1175/1520-0469(1949)006<0001:OEDITA >2.0.CO;2

Zhang, P., and Ni, Y. Q. (1991). The Effect of Topographic Forcing on the Formation and Maintenance of Blocking. Adv. Atmos. Sci. 8, 317-326. doi:10.1007/BF02919614

Zhu, Q., Lin, J., Shou, S., and Tang, D. (2007). Synoptic Principles and Methods. China Meteorological Press (In Chinese). 186-187.

Conflict of Interest: The authors declare that the research was conducted in the absence of any commercial or financial relationships that could be construed as a potential conflict of interest.
Publisher's Note: All claims expressed in this article are solely those of the authors and do not necessarily represent those of their affiliated organizations, or those of the publisher, the editors, and the reviewers. Any product that may be evaluated in this article, or claim that may be made by its manufacturer, is not guaranteed or endorsed by the publisher.

Copyright (c) 2022 Zhang, Li, Wang, Zhang and Lu. This is an open-access article distributed under the terms of the Creative Commons Attribution License (CC BY). The use, distribution or reproduction in other forums is permitted, provided the original author(s) and the copyright owner(s) are credited and that the original publication in this journal is cited, in accordance with accepted academic practice. No use, distribution or reproduction is permitted which does not comply with these terms. 